\title{
Percutaneous Biliary External-Internal Drainage for Malignant Obstruction of Self-Expanding Uncovered Metal Stents Placed for Hilar Carcinoma: Retrospective Analysis of Long-Term Outcome in 160 Patients
}

\author{
Didier H. Bonnel ${ }^{1}$ Alice Gangloff ${ }^{2} \quad$ Abe Fingerhut ${ }^{3,4}$
}

\author{
${ }^{1}$ Department of Biliary Interventional Radiology, Imagerie Médicale \\ Tourville, Paris, France \\ 2Department of Gastroenterology and Hepatology, CHU Charles-Nicolle, \\ Rouen, France \\ ${ }^{3}$ Department of Surgery, Section for Surgical Research, Medical \\ University of Graz, Graz, Austria \\ ${ }^{4}$ Department of Gastrointestinal Surgery, Ruijin Hospital, Shanghai Jiao \\ Tong University School of Medicine, Shanghai, China
}

Address for correspondence Didier H. Bonnel, MD, Department of Biliary Interventional Radiology, Imagerie Médicale Tourville, 19 avenue de Tourville, Paris 75007, France (e-mail: db@imagerietourville.com).

\begin{abstract}
Keywords

- percutaneous biliary stent

- bile duct malignancy

- hilar carcinoma

- biliary stent obstruction

Aims and Objectives We evaluated the safety and effectiveness of reestablishing adequate bile flow in patients with malignant obstruction of multilobar self-expanding uncovered metal stents (SEUMSs) inserted for palliative treatment of malignant hilar stenosis, an otherwise fatal setting. From September 1991 to January 2019, 160 patients (100 men and 60 women; median: 69 [range: $37-87$ ] years) with malignant obstruction of multilobar SEUMSs were treated by percutaneous transhepatic external-internal biliary drainage. All patients were followed in their respective oncology services where they were treated (or not) according to local protocols adapted to their disease and overall general status.

Result External-internal biliary drainage was successful in all 160 patients. All patients were discharged within 5 days. Both immediate (4 patients; $2.5 \%$ ) and late complications (29 patients; $18 \%$ ) were managed successfully with minimal morbidity and no mortality. After reestablishment of adequate bile flow, the median survival was 191 days (range: $2-5,668$ days). Forty-one (25.6\%) patients survived for more than 12 months, and $10(6.2 \%)$ survived for more than 3 years.

Conclusion In our series, external-internal biliary drainage was safe and effective; it was life-saving and afforded acceptable quality of survival for patients who otherwise would have been left untreated with short-term fatality.
\end{abstract}

\section{Introduction}

Hilar cholangiocarcinomas are nonresectable in 44 to $53 \%$ of patients. ${ }^{1,2}$ Likewise, for hilar metastases, which are nonresectable by definition, only palliative treatment can be envisioned. Currently, stents can be placed either endoscopically (duodenoscopy) or percutaneously (transhepatic biliary drainage) to relieve nonresectable malignant hilar obstruction. The percutaneous transhepatic route is indicated in case of failure or anatomical impossibility of endoscopic drainage or when such drainage is incomplete and infection of undrained territories occurs. The median overall duration of patency of self-expanding uncovered metal stents (SEUMSs) is 178 days (range: $128-180$ days) ${ }^{3}$; however, malignant obstruction of SEUMSs occurs in up to
DOI https://doi.org/

$10.1055 / \mathrm{s}-0039-3401902$

ISSN 2457-0214.
License terms

()(1) $\Theta \circledast$ 
$21 \%$ of patients who survive longer than the usual patency of such stents. ${ }^{4}$ In this setting, the short-term vital prognosis is threatened because of septic obstructive cholangitis. Revisional disobstruction of these stents is most often impossible endoscopically because of entangled metallic meshes at the hilum of the liver. In this situation, percutaneous transhepatic biliary drainage can be a salvage alternative. We report a series of 160 patients who presented with purulent cholangitis due to malignant obstruction of their SEUMSs placed for primary or metastatic hilar carcinoma treated with definitive percutaneous transhepatic external-internal drainage. The main goal was to determine whether this type of management could reverse the episode of life-threatening cholangitis and then ensure an acceptable quality of survival.

\section{Patients and Methods}

\section{Patients}

From September 1991 to January 2019, 160 patients (100 men and 60 women, with a median age of 69 years [range: 37-87 years]) having undergone multiple endoscopic, percutaneous transhepatic, or mixed endoscopic and percutaneous transhepatic insertion of SEUMSs for unresectable hilar malignancy, were referred to our institution for malignant obstruction of their biliary stents. Percutaneous transhepatic external-internal drainage was decided either after failed endoscopic management or when the endoscopic route was judged technically impossible, usually due to multiple entangled metallic meshes at the porta hepatitis.

The median delay between the initially placed SEUMSs and secondary neoplastic obstruction was 184 days (range: 5-4,363 days).

All patients had a histological report indicating carcinoma. The type of cancer is specified in - Table $\mathbf{1}$. We

Table 1 Type of malignancy

\begin{tabular}{|l|l|}
\hline Primitive carcinoma & 64 \\
\hline Cholangiocarcinoma & 11 \\
\hline Gallbladder carcinoma & 24 \\
\hline Pancreatic adenocarcinoma & 2 \\
\hline Hepatocellular carcinoma & 3 \\
\hline Pancreatic neuroendocrine tumor & 1 \\
\hline Ampullary carcinoma & 105 \\
\hline Total & \\
\hline Secondary cancers metastatic to the hilum & 35 \\
\hline Colorectal & 10 \\
\hline Gastric & 6 \\
\hline Mammary & 1 \\
\hline Uterine & 1 \\
\hline Urothelial & 1 \\
\hline Thyroid & 1 \\
\hline Lymphoma & 55 \\
\hline Total & 160 \\
\hline Overall total & \\
\hline &
\end{tabular}

used a simplified version ${ }^{5}$ of the de Oliveira classification ${ }^{6}$ to describe the location of obstruction (-Table 2 ). Of the 160 patients, 124 patients had type II stenosis, 36 had type III or greater than type III stenosis; among these, 20 had diffuse intrahepatic neoplastic infiltration of the biliary tree (neoplastic cholangitis; - Fig. 1).

Table 2 Simplified de Oliveira classification for neoplastic hilar stenosis ${ }^{8}$

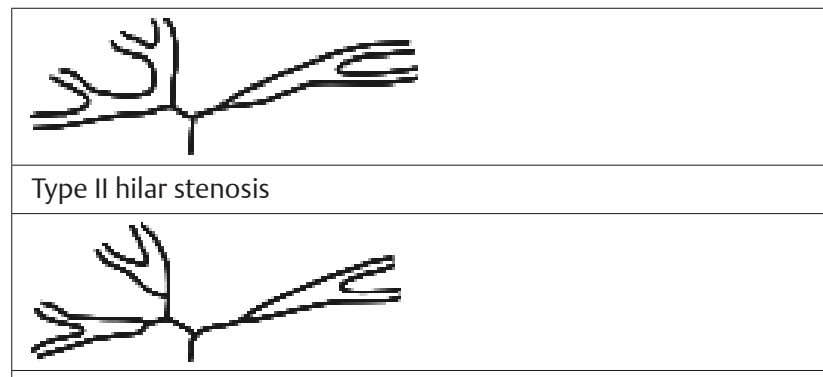

Type III hilar stenosis

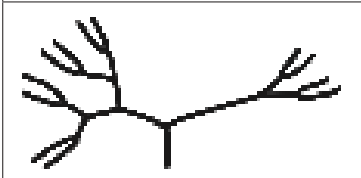

Greater than type III hilar stenosis

Anatomical classification of hilar stenoses: type II corresponds to stenosis located at the level of the main convergence and affects the right and left livers. This requires bilateral drainage; type III corresponds to stenosis affecting the main convergence with extension on the right reaching the anterior and posterior bile ducts, separating the liver into three sectors (anterior right, posterior right, and left). This situation requires a triple drainage. Disease involvement beyond type III usually does not allow complete drainage of all liver segments. These were grouped together and designated as "> III"; their treatment is univocal: find and drain the largest of the sectors.

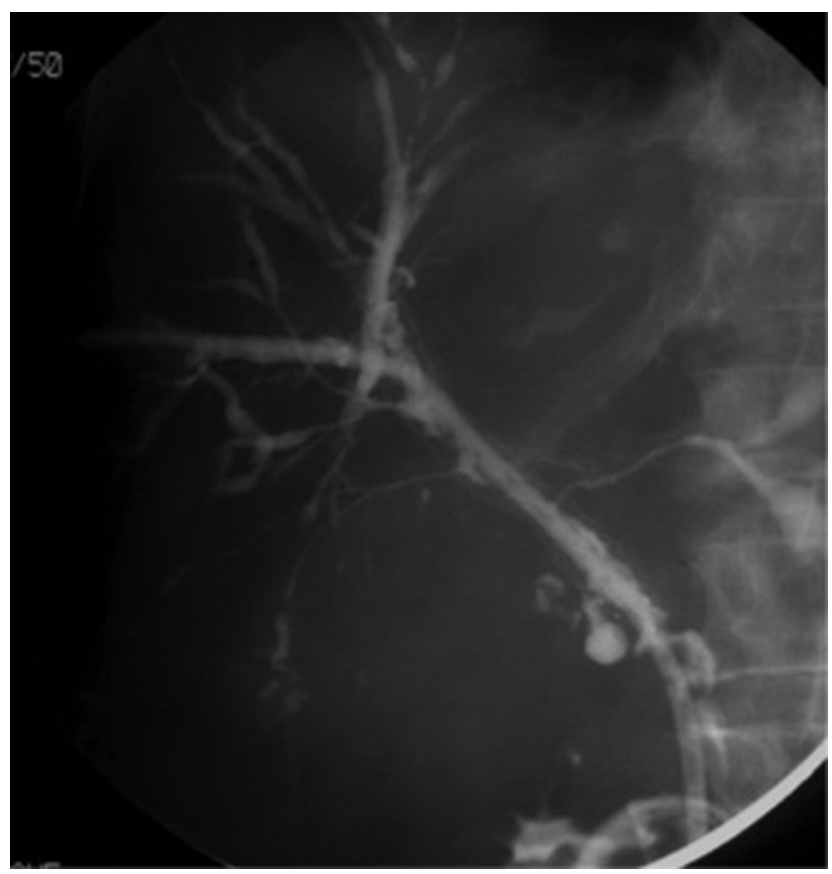

Fig. 1 Neoplastic cholangitis: diffuse neoplastic infiltration of the intrahepatic biliary tree. 
Initial palliative SEUMS insertion is detailed in - Table 3. Transhepatic stent insertion had been performed according to the recommendation of Nakayama et al. ${ }^{7}$ Complete drainage was defined as an absence of undrained intrahepatic biliary tree segments proximal to the biliary tract stenosis. With regard to the initial drainage, 140 had complete drainage and 20 had incomplete drainage due to neoplastic cholangitis; 124 patients had had bilateral drainage and 36 had three or more lateral or intersegmental drainage.

All patients were followed in their respective oncology services where they were treated (or not) according to local protocols adapted to their disease and overall general status.

\section{Methods}

All patients were hospitalized on the day of the procedure, which was performed under general anesthesia (neuroleptic analgesia). The patient was positioned supine, with the operator on the right, left, or successively on both sides according to the side of the bile duct drainage. The skin was prepared with $10 \%$ povidone-iodine solution (Betadine, Pfizer) and was draped accordingly to the planned transhepatic drainage. Single-dose first-generation $\beta$-lactam cephalosporin (Cefazolin $2 \mathrm{~g}$ ) was administered intravenously at anesthetic induction.

Because all the 160 patients had multiple entangled metallic meshes in the hilar region, the following steps were performed successively:

- Percutaneous transhepatic cholangiography to determine the level of obstruction.

- Insertion of hydrophilic guidewires (-Fig. 2) (Radifocus Guidewire M 0.035 [0.89 mm], Terumo Corporation) followed by insertion of coaxial dilatators (8/10 Dilatator/ Sheath Set, Boston Scientific) and angioplasty balloons (Mars PTA-Balloon Catheter, Optimed Inc.), and, finally, insertion of $14 \mathrm{~F}$ internal-external biliary drain (-Fig. 3a, b) (14F Biliary Drainage Catheter, Cook Inc.) to restore biliary patency.

Once the $14 \mathrm{~F}$ internal-external biliary drain(s) were placed, they were allowed to drain by dependency during 3 days. The drains were then clamped and the external segment was left under a simple dressing.

One or more drains were inserted according to whether left/right communication was patent or not (-Fig. 4).

Table 3 Initial palliative treatment according to the simplified de Oliveira classification

\begin{tabular}{|l|l|l|l|}
\hline & $\begin{array}{l}\text { Percutaneous } \\
\text { procedure }\end{array}$ & $\begin{array}{l}\text { Endoscopic } \\
\text { procedure }\end{array}$ & $\begin{array}{l}\text { Combined } \\
\text { procedure }\end{array}$ \\
\hline $\begin{array}{l}\text { Type II }(n=124) \\
\pm \text { neoplastic } \\
\text { cholangitis }\end{array}$ & 72 & 35 & 17 \\
\hline $\begin{array}{l}\text { Type III or >III } \\
(n=36) \\
\pm \text { neoplastic } \\
\text { cholangitis }\end{array}$ & 0 & 21 & 15 \\
\hline
\end{tabular}

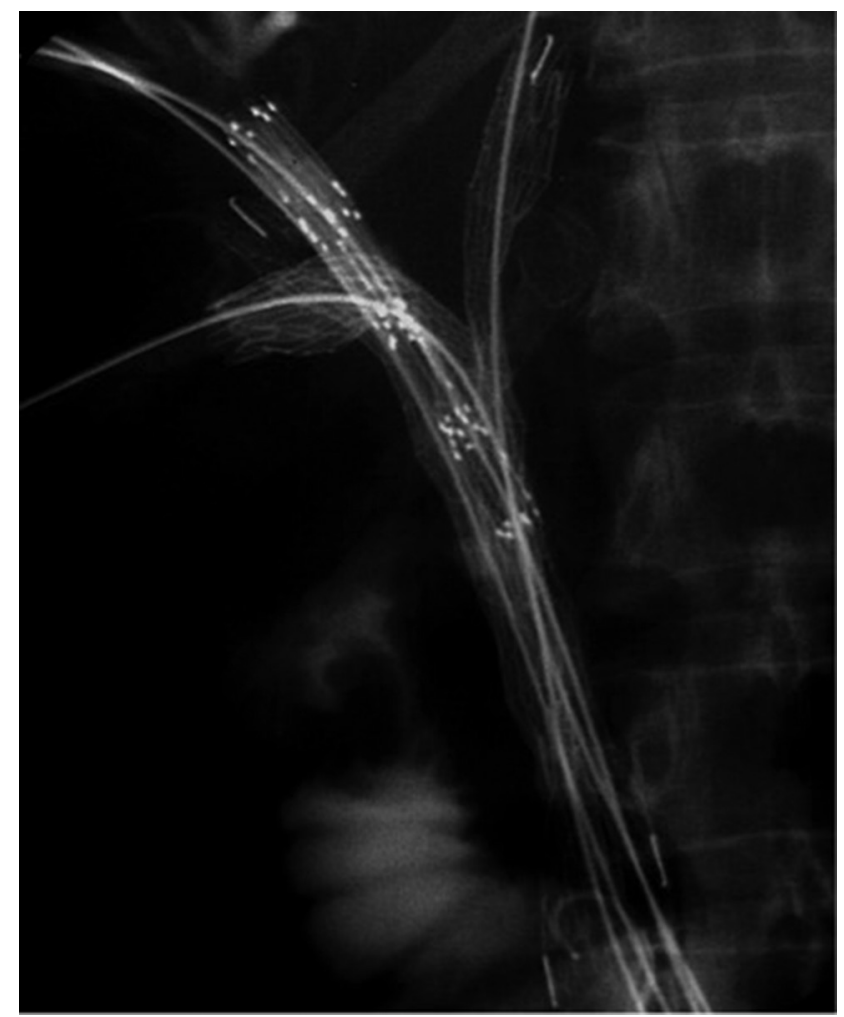

Fig. 2 Insertion of the hydrophilic guidewire through the meshes of the metallic stents and through the stenosis.

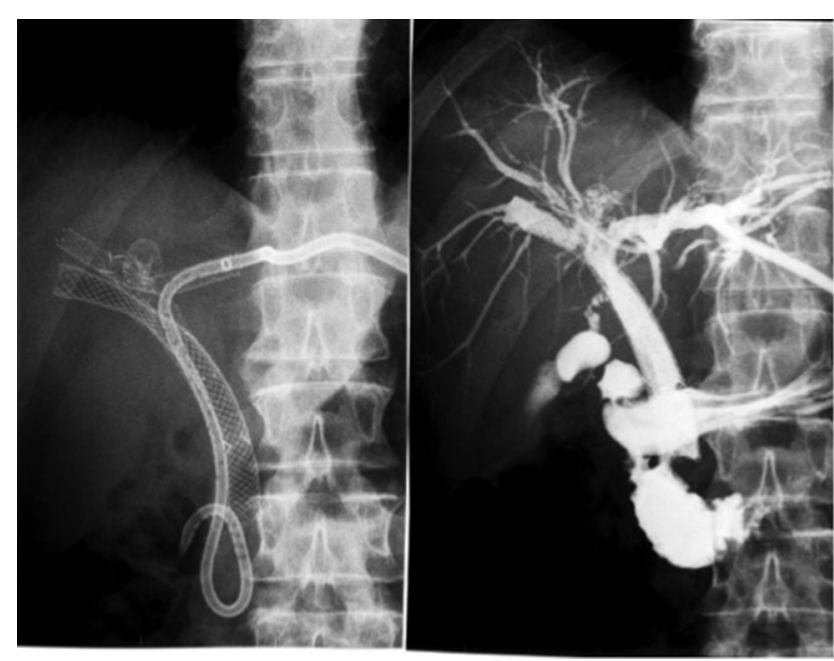

Fig. 3 Triple transhepatic approach due to intrahepatic extension of stent obstruction.

Patients were allowed to leave the hospital on day 5 in the absence of recurrent sepsis or leakage around the drain.

After patient discharge, the patient, family, and paramedical and medical circle were informed by a written document that in case of cholangitis or bile leakage, the drain should be unclamped and allowed to drain by dependency into an adapted recipient bag and the patient be referred for rehospitalization.

The drains were changed prophylactically every 4 to 6 weeks to avoid plugging and recurrent cholangitis, under general anesthesia in an ambulatory unit. Leaks around the 


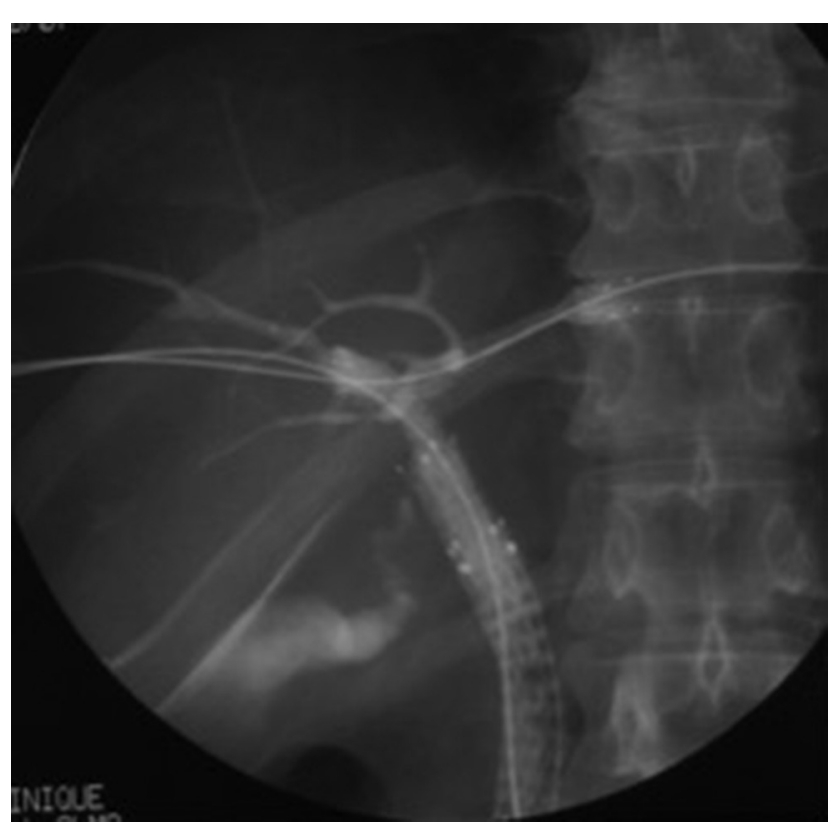

Fig. 4 Insertion of a $14 \mathrm{~F}$ internal-external biliary drain to relieve the obstruction of multilobar hilar self-expanding uncovered metal stent (SEUMS). (a) The 14F internal-external biliary drain in place. (b) A complete drainage of the liver postdie injection, thanks to the previously introduced SEUMS.

drain were classified into three grades according to their quantity: grade I, bile leaks that only stained the dressing; grade II, bile leaks that seeped through the dressing; grade III, bile leaks that not only seeped through the dressing but also stained the patients' clothing and/or bedding. Only grade II and III leaks required repositioning of the biliary catheter in an ambulatory unit. The dressing was changed every day either by a home nurse or the patient's advocate attending the patient.

Patient data and outcomes were recorded prospectively but analyzed retrospectively, and the article was written according to the STROBE (Strengthening the Reporting of Observational Studies in Epidemiology) guidelines. The success rate of placing drains through the interstices of the metallic prosthesis, the nature and number of complications related to the procedure, to the drain itself, and the duration and quality of survival were recorded and analyzed at the closing date, that is, January 20, 2019.

\section{Results}

Transhepatic cholangiography detected endoluminal tumoral budding within the SEUMSs in all 160 patients. The level of malignant obstruction was below the main biliary convergence (therefore without interruption of communication between the right and the left hemilivers) in 151 patients, whereas in 9 patients, obstruction was located above the main biliary right/left liver confluent. In the former, adequate drainage required only one drain ( $\boldsymbol{- \text { Fig. }} \mathbf{3 a}, \mathbf{b}$ ). In the other nine patients with disconnected right and left hemilivers, two drains were necessary to achieve adequate drainage.

Immediate complications were observed in four $(2.5 \%)$ patients: hemobilia, which was treated with success by hepatic arterial embolization (three patients) and bile pleural effusion, which was treated with success by simple pleural drainage (one patient). None of these immediate complications were life-threatening.

Late complications were observed in 29 (18\%) patients: 18 patients had grade II or III bile leaks around the drain that required repositioning associated or not with additional lateral drainage holes made in the drain. Eleven patients had one leak episode, three had two episodes, two had four episodes, and one patient each had five and seven episodes, respectively. Five patients lost their drain and required reinsertion through the percutaneous transhepatic tract. Two patients had recurrent cholangitis due to drain obstruction that required an early drain change. Two patients had portal venous bleeding exteriorized by their drain when the drain eroded a portal vessel requiring repositioning of the drain to remove the lateral hole from the portodrain communication. One patient sustained an abscess around the skin incision. One patient had acalculous acute cholecystitis treated by percutaneous cholecystostomy. These late complications led to 38 rehospitalizations.

After bile flow was reestablished, the median survival was 191 days (range: 2-5,668 days). Forty-one (25.6\%) patients survived for more than 1 year, and $10(6.2 \%)$ survived for more than 3 years ( - Table 4 ).

\section{Discussion}

In this series, successful and definitive salvage transhepatic drainage of patients with malignant obstruction of SEUMSs previously inserted for the palliative treatment of malignant hilar stenosis was achieved in all 160 patients. All patients had type II or higher hilar stenosis (according to the simplified de Oliveira anatomical classification ${ }^{5}$; - Table 2). All patients had been referred for life-threatening cholangitis after failed or impossible salvage endoscopic treatment of obstruction.

In spite of the technical difficulties related to entangled metallic meshes in the hilar region, we believe that our 100\% success rate could be attributed to the particular technical

Table 4 Duration of survival and type of carcinoma in the 10 patients who survived for more than 3 years

\begin{tabular}{|l|l|}
\hline $\begin{array}{l}\text { Duration of survival: (days) } \\
\text { years, months, weeks }\end{array}$ & Type of carcinoma \\
\hline$(5,668) 15 \mathrm{y}$ and $6 \mathrm{mo}$ & Cholangiocarcinoma \\
\hline$(3,827) 10 \mathrm{y}$ and $6 \mathrm{mo}$ & Colonic carcinoma \\
\hline$(3,699) 10 \mathrm{y}$ and $7 \mathrm{wk}$ & Cholangiocarcinoma \\
\hline$(3,433) 9 \mathrm{y}$ and $4 \mathrm{mo}$ & Cholangiocarcinoma \\
\hline$(2,831) 7 \mathrm{y}$ and $9 \mathrm{mo}$ & Cholangiocarcinoma \\
\hline$(1,903) 5 \mathrm{y}$ and $10 \mathrm{wk}$ & Cholangiocarcinoma \\
\hline$(1,480) 4 \mathrm{y}$ and $1 \mathrm{wk}$ & Cholangiocarcinoma \\
\hline$(1,221) 3 \mathrm{y}$ and $5 \mathrm{mo}$ & Colonic carcinoma \\
\hline$(1,168) 3 \mathrm{y}$ and $3 \mathrm{mo}$ & Cholangiocarcinoma \\
\hline$(1,107) 3 \mathrm{y}$ and $1 \mathrm{wk}$ & Colonic carcinoma \\
\hline
\end{tabular}


steps outlined previously. The fact that 41 (25.6\%) patients in our series survived longer than 1 year and 10 (6.2\%) longer than 3 years attests to the safety and durability associated with our technique.

In our series, the median delay before neoplastic obstruction of initial SEUMSs was 184 days (range: 5-4,363 days), remarkably close to the median overall duration of patency of SEUMSs for neoplastic obstruction found in the literature (178 days). ${ }^{3}$ In our practice, SEUMSs are preferred over plastic prostheses due to longer patency. ${ }^{8,9}$ Although no statistically significant difference has been found in patency compared with uncovered stents, ${ }^{10}$ covered stents are not indicated in hilar obstructions because they do not allow leftright lobe communication, as do stents with open meshes. In this setting, we chose to insert an external-internal biliary drain rather than a new stent to ensure long-term definitive drainage because in case of eventual reobstruction, additional metallic stents across the hilum would increase the complexity of drainage maneuvers and surely in some cases result in failure of the internal drainage. This would lead to severe alteration of patients' quality of life due to the lifelong necessity of external drainage and a collection bag.

In spite of extensive research, we were unable to find any other series in the literature proposing long-term management for the malignant obstruction of multiple SEUMSs previously inserted for palliative treatment of hilar (type II and higher) malignant stenosis when further endoscopic procedures are no longer possible. It is therefore probable that in most centers, these patients are considered hopeless and that further treatment is futile. Our results go against this attitude because a substantial percentage of patients in our series had a comfortable, complication-free, and sometimes prolonged survival. We were able to find only one series on percutaneous salvage management for recurrent malignant metallic stent obstruction, but this concerned stent occlusion in patients with type I stenosis, who are much easier to treat than the patients in our series who had type II or higher stenoses. ${ }^{11}$ The only other series concerning obstructed stents in type II or higher malignant biliary obstruction was a retrospective study on endoscopic management of 52 patients. ${ }^{12}$ The $90 \%$ rate of clinical success reported in this series was high, attesting to expert retrograde management, but immediate relief was short-lived (68-day mean delay to reobstruction). However, the results of this series ${ }^{12}$ call for several remarks. First, only patients with bilateral stents were included, meaning that we do not know if this treatment modality would work for trilateral or intersegmental stents (36 patients in our series). Second, the 68-day mean delay to reobstruction can be considered to be short. Third, the authors did not propose any solution for the treatment of reobstruction or raise the problem of compounding the complexity of drainage technique by introducing new metallic meshes in the region of the hilum. The advantage of our technique is that it can be used in patients irrespective of the type of hilar stenosis and the number of metallic stents crossing the hilum already in place. Moreover, our technique allows for simple routine periodical replacement of the $14 \mathrm{~F}$ biliary catheter, which would not have been the case if additional metallic prosthesis were inserted to treat stent obstruction.

Of note, the 151 (94\%) patients who had secondary obstruction below the convergence were salvaged with a single biliary drain, with obvious benefits in terms of comfort for their remaining survival. Preserved intrahepatic communication due to previously placed and patent intrahepatic SEUMSs ( $\mathbf{- F i g}$. 3a,b) was somewhat a surprise and does not seem to have been reported before in the literature. The high prevalence of obstruction below the convergence with preserved intrahepatic communication and our 100\% success rate plead in favor of vigorous treatment procedures for the malignant obstruction of SEUMSs for these otherwise short-term compromised patients presenting with hilar carcinomas.

Our 2.5\% nonlethal immediate complication rate was low considering the clinical severity of patient status in our series.

The long-term tolerance of external-internal drainage in our series, which constitutes an argument in favor of proposing a poised interventional policy, was assessed as follows. First, patient acceptance was high, and most preferred the presence of one or eventually two external-internal drains and repeat outpatient visits for drain replacement to multiple sessions of traditional endoscopic or percutaneous drainage (that all of these patients had had to endure for the initial palliative treatment of their malignant hilar obstruction). Second, the quality of survival of patients undergoing interval palliative treatment for terminal disease could be considered as acceptable (131 [82\%] patients in our series never had any drain complication or malfunction that required rehospitalization).

In our series, 29 (18\%) patients required 38 rehospitalizations for drainage problems. Of note, a nonoperative solution was found for all patients with early or late complications, even in the case of bleeding (percutaneous embolization) or acalculous acute cholecystitis (percutaneous cholecystostomy). This attests to the safety of the procedure.

The consequence of routine prophylactic changing of the drain every 4 to 6 weeks, leading to direct and regular contact between the patients and the interventional radiologist, was that no patient in this series was lost to follow-up. All deaths in our series were due to on-going disease, not the palliative procedure. The fact that $41(25.6 \%)$ patients in our series had a survival of at least 1 year (31 patients lived for more than 12 months, 10 lived for more than 3 years) is indeed surprising considering the severity of their disease. With regard to patients who had lived for more than 3 years (-Table 4), efficacy of chemotherapy in three patients who had colorectal hepatic metastases could be an explanation, whereas for the seven other patients who had been categorized as having "histologically proven cholangiocarcinoma," we cannot exclude the possibility of misdiagnosis as confusion with sclerosing cholangitis has been reported. ${ }^{13}$

Our study has several limitations. This was a retrospective study, a single operator's (DHB) experience over a long time span (27 years and 3 months) of patients who were referred from other institutions. 


\section{Conclusion}

Malignant obstruction of SEUMSs inserted for the palliative treatment of hilar malignancy is a life-threatening situation due to the short-term septic consequences of obstructive cholangitis. No other formal therapeutic plan has been proposed until now. The excellent outcome in our series of 160 patients encourages us to propose this therapeutic modality for these patients who otherwise, most likely, would have succumbed to ensuing sepsis. Moreover, the acceptable quality and sometimes prolonged survival of these patients is another argument in favor of vigorous management in this setting.

\section{Funding}

None.

\section{Conflict of Interest}

None.

\section{Acknowledgment}

The authors would like to thank Mrs. Fabienne Poinsot, Head Secretary of the Department of Biliary Interventional Radiology, Imagerie Médicale Tourville, for updating the patient records and collecting data.

\section{References}

1 Molina V, Sampson J, Ferrer J, et al. Klatskin tumor: diagnosis, preoperative evaluation and surgical considerations. Cir Esp 2015;93(9):552-560

2 Kawasaki S, Imamura H, Kobayashi A, Noike T, Miwa S, Miyagawa S. Results of surgical resection for patients with hilar bile duct cancer: application of extended hepatectomy after biliary drainage and hemihepatic portal vein embolization. Ann Surg 2003;238(1):84-92

3 Hong W, Zhu Y, Dong Y, Wu Y, Zhou M, Ni H. Predictors for occlusion of the first inserted metallic stent in patients with malignant biliary obstruction. Saudi J Gastroenterol 2015;21(6):386-390

4 Lee $\mathrm{BH}$, Choe DH, Lee JH, Kim KH, Chin SY. Metallic stents in malignant biliary obstruction: prospective long-term clinical results. AJR Am J Roentgenol 1997;168(3):741-745

5 Bonnel D, André T, Mader B, Lefebvre JF, Bensoussan E, Liguory $C$. Malignant biliary obstruction, general review and clinical practice [in French]. Bull Cancer 2013;100(5):443-452

6 Deoliveira ML, Schulick RD, Nimura Y, et al. New staging system and a registry for perihilar cholangiocarcinoma. Hepatology 2011;53(4):1363-1371

7 Nakayama T, Ikeda A, Okuda K. Percutaneous transhepatic drainage of the biliary tract: technique and results in 104 cases. Gastroenterology 1978;74(3):554-559

8 Sawas T, Al Halabi S, Parsi MA, Vargo JJ. Self-expandable metal stents versus plastic stents for malignant biliary obstruction: a meta-analysis. Gastrointest Endosc 2015;82(2):256-267.e7

9 Moole H, Jaeger A, Cashman M, et al. Are self-expandable metal stents superior to plastic stents in palliating malignant distal biliary strictures? A meta-analysis and systematic review. Med J Armed Forces India 2017;73(1):42-48

10 Li J, Li T, Sun P, Yu Q, Wang K, Chang W, Song Z, Zheng Q Covered versus uncovered self-expandable metal stents for managing malignant distal biliary obstruction: a meta-analysis. PLos One 2016;11(2):e0149066

11 Cwikiel W. Percutaneous management of occluded biliary duct endoprostheses. Acta Radiol 2000;41(4):338-342

12 Inoue $\mathrm{T}$, Naitoh I, Okumura $\mathrm{F}$, et al. Reintervention for stent occlusion after bilateral self-expandable metallic stent placement for malignant hilar biliary obstruction. Dig Endosc 2016;28(7):731-737

13 Lazaridis KN, Gores GJ. Primary sclerosing cholangitis and cholangiocarcinoma. Semin Liver Dis 2006;26(1):42-51 